\title{
Rechtsverwirklichung als Chefsache
}

\author{
C. A. Zenger
}

Zwei Irrtümern sollte man nicht erliegen: Nämlich, dass das Recht am besten ausgesperrt werden sollte, um gute Medizin betreiben $\mathrm{zu}$ können, und dass die Rechtsnormen objektiv feststehen und sich wie in einem Rezeptbuch nachlesen lassen.

Zum einen ist die Tätigkeit des Arztes und des Spitals in vielfältige rechtliche Beziehungen eingebettet, die sie stützen und entlasten; so selbstverständlich ist diese «rechtliche Atmosphäre», dass man sie im Alltag kaum wahrnimmt. Zum andern sind Gesetzes- oder Verordnungsbestimmungen auslegungsbedürftig und lassen zum Teil beträchtliche Beurteilungs- und Entscheidungsspielräume offen, welche die Behörden, aber auch die Beteiligten ausfüllen (selbst wenn sie das gar nicht realisieren), und überdies können die Beteiligten durch vertragliche Vereinbarung selbst Recht setzen, das zwischen den Vertragspartnern gilt.

Beides hat grosse praktische Bedeutung; denn wer im Gesundheitswesen tätig ist, ist einerseits in rechtlich bestimmte Verhältnisse und Beziehungen eingebunden, beeinflusst aber andererseits durch seine Entscheidungen das gelebte Recht. Die in der Praxis tätigen Ärzte und Spitäler prägen den Inhalt des Rechts wohl mindestens ebenso intensiv wie Berufs- und andere Interessenverbände, welche sich die politische Einflussnahme auf die Fahnen geschrieben haben.

Der Einfluss eines Spitals auf den Inhalt des Rechts ist um einiges grösser als der eines einzelnen Arztes. Er wirkt im Aussenverhältnis zwischen dem Spital und seinem gesundheitspolitischen Umfeld, beispielsweise bei der Entwicklung des Leistungsauftrags und der Sicherung der Finanzierung, beim Einsatz von Heilmitteln, bei Massnahmen zur Krankheitsverhütung oder der Eindämmung von Gesundheitsrisiken. Er entfaltet seine Wirkungen aber auch im Innenverhältnis zwischen dem Spital und seinen Prozessen, etwa bei der Erstellung von Stellenprofilen, der Handhabung von Dienstverhältnissen, der Patientenaufklärung, der Prävention von Behandlungsfehlern, der Sicherung und Geheimhaltung von Personendaten, der Entscheidung über Sicherungs- und Zwangsmassnahmen oder der betrieblichen Gesundheitsförderung. Und nicht zuletzt beeinflusst das Spital auch das Handeln des einzelnen Arztes, beispielsweise bei Behand- lungsentscheiden am Lebensanfang und am Lebensende, bei der Ablehnung von Behandlungsmassnahmen durch Patienten, bei medizinischen Forschungsuntersuchungen, bei Rationierungsentscheiden oder im Umgang mit psychisch oder sozial schwierigen Patienten.

Dieser Einfluss sollte nicht zufällig, sondern zielgerichtet sein. Um so wichtiger ist, dass Spitalleitungen die rechtlichen Gestaltungsspielräume und ihre Grenzen kennen. Aber nicht nur das: Sie müssen vor allem dafür sorgen, dass rechtliche Regeln im Betrieb verwirklicht und die Spielräume ausgefüllt werden. Dafür müssen sie die nötigen organisatorischen Bedingungen schaffen; denn Recht wird im Spitalbetrieb nicht primär durch Verhaltensvorschriften verwirklicht. Das Verhalten der einzelnen Personen lässt sich durch Weisungen nur sehr begrenzt steuern, und der gute Wille des Personals allein nützt nichts. Entscheidend ist, dass Strukturen und Prozesse organisatorisch so ausgestaltet werden, dass sie rechtstreues Verhalten erzwingen oder wenigstens nützlich erscheinen lassen. Die Rechtsverwirklichung im Spitalbetrieb ist in erster Linie eine Frage der Organisation, die Verantwortung dafür liegt bei der Führung.

Medizinische Leitungen nehmen sich heute der Aufgaben der Rechtsverwirklichung oft nur ganz am Rand an. Weil sie sich nicht dafür engagieren, übernehmen die Verwaltungsleitungen das Ruder. Die Strukturen und Prozesse des Spitals, in welche die medizinische Tätigkeit eingebunden ist, sind deshalb zunehmend nach betriebswirtschaftlichen Vorstellungen organisiert. Daran kann sich nur dann etwas ändern, wenn auch die medizinischen Leitungen Verantwortung für die Rechtsverwirklichung übernehmen. Diese müssen garantieren, dass der medizinische Behandlungsauftrag, der rechtlich an erster Stelle steht, erfüllt werden kann und die Rechte der Patienten gewahrt werden. Chefärzte brauchen deswegen nicht auch noch Juristen zu werden; es genügt, dass sie die Funktionen des Rechts verstehen und seine Schlüsselkonzepte kennen. Juristische Fachkompetenz soll ihnen die Spielräume und Grenzen zeigen und ihre inhaltlichen Vorstellungen in rechtliche Formen giessen. Die inhaltlichen Vorstellungen zu entwickeln aber ist nicht nur Chefsache, sondern auch Sache des Chefarztes. 


\section{Rechtliche Leitplanken im Spital}

\section{Fortbildung in Managementfragen für Ärztinnen und Ärzte in leitender Stellung}

Hintergrund

Die Umstrukturierungen in den Spitälern (Spitalverbünde, Allianzen von öffentlichen und privaten Spitälern usw.) stellen Chefärzte und leitende Ärzte vor neue rechtliche Herausforderungen. Gleichzeitig gewinnen Fragen der Haftung, der Anstellungs- und Arbeitsbedingungen, der Lehren aus Critical Incidents usw. immer grössere Bedeutung im Alltag der Klinikführung. Mit den juristischen Leitplanken vertraut zu sein, gehört heute zum unverzichtbaren Rüstzeug eines Arztes in leitender Funktion und gibt ihm die nötige Sicherheit.

\section{Kursinhalt}

Juristische Aspekte der Umstrukturierungen von Spitälern, Veränderungen durch New Public Management und Privatisierung. Rechte und Pflichten eines Arztes in leitender Stellung. Juristische Aspekte der medizinischen Behandlungen, der Patientenaufklärung und -einwilligung, Umgang mit Haftungsfällen und Lehren aus Critical Incidents usw. Spielräume und Grenzen für rechtlich und ethisch-politisch vertretbare Entscheidungen.

\section{Lernziel}

Die Teilnehmenden kennen die wichtigsten rechtlichen Leitplanken für die chefärztliche Tätigkeit. Sie verstehen, dass das Recht einen Rahmen für die medizinische Behandlung bildet, der Grenzen setzt, aber auch entlastend wirkt. Sie erkennen die Entscheidungsspielräume und die ethisch-politische Verantwortung des Arztes in leitender Stellung innerhalb dieses Rahmens.

\section{Weitere Informationen und Anmeldung}

Methoden: Fallstudien, Kurzvorträge, eigene Fallbeispiele der Teilnehmenden, Diskussion.

Kursleiter: RA Dr. Christoph Andreas Zenger, LL.M., Dozent an der Universität Bern.

Datum: 5./6. Juni 2003.

Kursort: Studienzentrum Gerzensee.

Kosten: 2 Tage inklusive Kursunterlagen: Fr. 1900.-. Zusätzlich Kosten für Unterkunft und Verpflegung. Aus didaktischen Gründen ist die Teilnehmerzahl auf 16 beschränkt.

Anmeldung bis spätestens 19. Mai 2003 an: zenger \& biaggi, Frau C. Baeriswyl, Postfach 418, 3074 Muri, Tel. 03195172 34, E-Mail: jean.biaggi@bluewin.ch. 